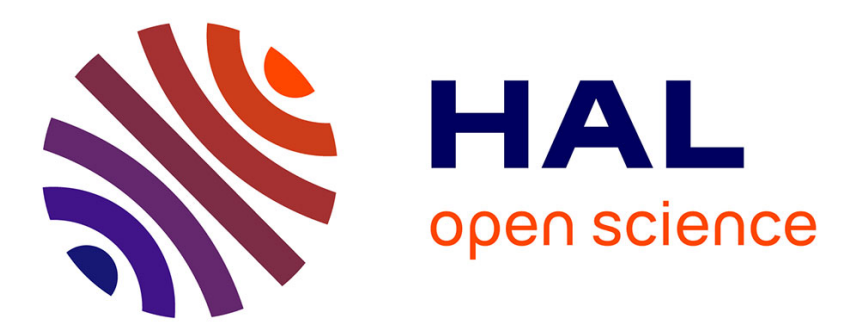

\title{
Optimal Reset Unknown Input Observer Design for Fault and State Estimation in a Class of Nonlinear Uncertain Systems
}

Iman Hosseini, Mirko Fiacchini, Paknoush Karimaghaee, Alireza Khayatian

\section{- To cite this version:}

Iman Hosseini, Mirko Fiacchini, Paknoush Karimaghaee, Alireza Khayatian. Optimal Reset Unknown Input Observer Design for Fault and State Estimation in a Class of Nonlinear Uncertain Systems. Journal of The Franklin Institute, 2020, 357 (5), pp.2978-2996. 10.1016/j.jfranklin.2019.12.008 hal03014495

\section{HAL Id: hal-03014495 \\ https://hal.univ-grenoble-alpes.fr/hal-03014495}

Submitted on 19 Nov 2020

HAL is a multi-disciplinary open access archive for the deposit and dissemination of scientific research documents, whether they are published or not. The documents may come from teaching and research institutions in France or abroad, or from public or private research centers.
L'archive ouverte pluridisciplinaire HAL, est destinée au dépôt et à la diffusion de documents scientifiques de niveau recherche, publiés ou non, émanant des établissements d'enseignement et de recherche français ou étrangers, des laboratoires publics ou privés. 


\title{
Optimal Reset Unknown Input Observer Design for Fault and State Estimation in a Class of Nonlinear Uncertain Systems
}

\author{
Iman Hosseini ${ }^{a}$, Mirko Fiacchini ${ }^{b}$, \\ Paknoush Karimaghaee ${ }^{a}$, Alireza Khayatian ${ }^{a *}$ \\ ${ }^{a}$ Shiraz University, Shiraz, Iran \\ ${ }^{b}$ Univ. Grenoble Alpes, CNRS, GIPSA-lab, 38000, Grenoble, France \\ * Corrsponding author: Khayatia@shirazu.ac.ir, 71348-51154,Shiraz University, \\ Shiraz, Iran
}

\begin{abstract}
This paper proposes a novel kind of Unknown Input Observer (UIO) called Reset Unknown Input Observer (R-UIO) for state and fault estimation of a class of nonlinear uncertain systems using linear matrix inequality (LMI) techniques. In the devised R-UIO, the states of the observer are reset to the after-reset value based on an optimal $H_{\infty}$ reset law in order to decrease the $\mathcal{L}_{2}$ norm and settling time of estimation error. It is shown that the utilization of such an observer can significantly improve the transient response of the observer. Moreover, the devised approach can be applied to both SISO and MIMO systems. Furthermore, the robust stability analysis of the devised R-UIO is addressed. Finally, the capabilities of the proposed method are demonstrated by applying it to a Continuous Stirred-Tank Reactor (CSTR) as a practical model.
\end{abstract}

Keywords - Reset Theory, Unknown Input Observer, Stability Analysis, State Estimation, Fault Estimation 


\section{Introduction}

Observer design is an attractive research field due to its importance in many practical applications such as observer-based control, fault diagnosis and fault tolerant control. In those areas, the problem of simultaneous state and fault estimation is very appealing since it has the capability of providing the required information about state and fault within one design.

Most of the practical systems include unknown inputs such as the parameter perturbation [1], actuator faults, and external disturbance [2]. All of the mentioned problems can be viewed as unknown inputs (UI) in industrial process, which can degrade the performance. Therefore, the problem of state and fault estimation of systems with UIs is very important and it is still an open problem. One of the widely used methods to tackle this problem is unknown input observer (UIO) design which has attracted many attentions since the past decades especially in the fields of observer-based control [3, 4], observer-based fault detection and isolation [2, 5].

In [6] and [7] an observer for linear systems subject to unknown inputs is developed. Besides, UIO design is investigated in [8] for linear non-minimum phase systems. The existence of a UIO is investigated in [9, 10], and the necessary and sufficient conditions for it are presented. Besides, the reduced order UIO can be designed using a systematic procedure [11, 12]. The capabilities of the UIOs for state and fault estimation in the presence of uncertainty and disturbance are demonstrated in [13, 14] and different approaches for designing an UIO have been developed. In [15], linear matrix inequalities are used to design a full-order nonlinear UIO for a class of nonlinear Lipschitz systems with unknown input. Moreover, a reduced order UIO for the one-sided nonlinear Lipschitz system is proposed in [16].

The performance of UIO is affected negatively by the modeling errors, parameter variations or other uncertain factors. Due to presence of uncertainty in the model of a physical system for designing a UIO, it is necessary that the designed UIO is robust against the uncertain factors and disturbance. Considering the factors, robust UIO is designed for unknown inputs TakagiSugeno models [17, 18] and linear parameter varying (LPV) system [19]. In [14, an LMI approach is used to design robust UIO for linear systems and a class of nonlinear systems. A robust UIO for fault detection using linear parameter varying model with uncertainties is presented in [20].

On the other hand, several control strategies are developed for dynamical systems in the past decades. However, most of them suffer from having oscil- 
latory transient responses [21]. In order to mitigate this issue and overcome the fundamental limitations of linear controllers, the idea of reset control theory can be utilized. In this theory, the states of the controller can be reset to a proper value, named after-reset value, based on an appropriately defined reset law. The idea of reset control originates from the Clegg Integrator which is aimed at tackling the drawbacks of the traditional integrators [22]. Based on this idea, the First Order Reset Element is developed [23]. In 24, the authors used the state-space representation rather than transfer functions and since then on, this control design method turns out to be an attractive field especially for practical applications [25, 26].

The stability and performance of the reset control systems are investigated in [27, 28]. The existence and uniqueness of solutions based on the well-posedness of reset instants are demonstrated in [29] and the necessary and sufficient conditions for them are provided. Moreover, reset systems stability has been used to check the global exponential stability of sample data systems [30]. In [31], state reset is used to stabilize switched linear singular systems. A similar idea is used in [32] for robust control and fault detection for these systems. A robust reset control law for systems with Lipschitz nonlinearity is designed in [33]. In [34], quadratic and exponential stability for the systems with saturation and nested saturations are investigated. In [35], an optimal adaptive reset control is used to enhance the transient performance and error bounds. A model predictive reset strategy is used to design a reset control for polytopic LPV systems, in [36].

Similarly, a traditional observer can change to a reset observer by utilizing the reset mechanism. A reset observer is a nonlinear observer consisting of a base observer and a reset law that resets the states of the observer when some predefined reset conditions are satisfied. In [37], a new type of adaptive observer is proposed by applying the reset to the observer states. In [38], an optimization problem is solved to obtain an optimal reset adaptive observer. In [39], the application of reset strategy to a proportional-integral observer for fault estimation problem is investigated. In [40] reset unknown input observer for linear systems is designed. In [41] reset proportional-integral observer for time-varying dynamics is developed.

In this paper, a class of nonlinear uncertain systems is considered and reset strategy is extended to the UIO to form a novel sort of UIOs called Reset UIO. An optimal after-reset value along with a proper jump sector is obtained by solving $H_{\infty}$ optimization problem. Furthermore, the stability analysis for the reset error dynamics is given. In addition, unlike most of the 
previous reset observers, the proposed approach can be applied to the MIMO systems as well as SISO systems. Moreover, the problem of fault estimation using the devised method is investigated. In this regard, an augmented system is constructed firstly and then the R-UIO for the augmented system is designed. The efficiency of the method is demonstrated by exploiting a CSTR as a practical example. It has been shown that exploiting the reset mechanism in the UIO can improve the performance of the observer in the sense of accuracy and rapidity.

The remainder of the paper is organized as follows: in Section 2, a conventional approach to design the base UIO is investigated. In Section 3. optimal reset UIO for state estimation is designed. In Section 4 the problem of simultaneous state and fault estimation is considered. In Section 5, the application of the proposed method to a practical model is presented and the results are compared with literature to validate the performance of the proposed estimation strategy. Finally, the concluding remarks are provided in Section 6.

\section{Conventional UIO (C-UIO)}

Consider the following uncertain nonlinear system:

$$
\begin{aligned}
& \dot{x}=(A+\Delta A) x+(B+\Delta B) u+D v+g(x) \\
& y=C x
\end{aligned}
$$

where $x \in \mathbb{R}^{n}, u \in \mathbb{R}^{m}, v \in \mathbb{R}^{d}$ and $y \in \mathbb{R}^{p}$ are the state vector, known input vector, unknown input vector and output of the system respectively. $A, B, C$ and $D$ are known matrices with appropriate dimension. Without loss of generality, it is assumed that $D$ is of full column rank [9]. $g(x)$ is a nonlinear function and the matrices $\Delta A$ and $\Delta B$ are time-varying matrices corresponding to uncertainty of nominal system. The following assumptions are also used throughout:

Assumption 1. $g(x)$ is locally Lipschitz on a domain (open and connected set) $\mathcal{D} \subset \mathbb{R}^{n}$ if each point of $\mathcal{D}$ has a neighborhood $\mathcal{D}_{0}$ such that $g(x)$ satisfies

$$
\|g(x)-g(\hat{x})\| \leq \gamma\|x-\hat{x}\|, \quad \forall x, \hat{x} \in \mathcal{D}_{0}
$$


Assumption 2. The vector space of the uncertainty matrices can be defined as follows:

$$
\begin{gathered}
\Gamma_{1}=\left\{\Delta A \mid \Delta A=T_{a} \Sigma_{a} N_{a}, \forall \Sigma_{a} \in R^{p_{a} \times q_{a}} \text { s.t. } \Sigma_{a}^{T} \Sigma_{a} \leq I\right\} \\
\Gamma_{2}=\left\{\Delta B \mid \Delta B=T_{b} \Sigma_{b} N_{b}, \forall \Sigma_{b} \in R^{p_{b} \times q_{b}} \text { s.t. } \Sigma_{b}^{T} \Sigma_{b} \leq I\right\}
\end{gathered}
$$

for appropriate values of $p_{a}, q_{a} \in N$ and matrices $T_{a}, T_{b}, N_{a}$ and $N_{b}$.

For the state estimation of the aforementioned system a full-order C-UIO can be defined in a way such that the observer leads to robustness against the uncertainties. Consider the UIO dynamics as

$$
\left\{\begin{array}{l}
\dot{z}=N z+G u+L y+M g(\hat{x}) \\
\hat{x}=z-E y \\
\hat{y}=C \hat{x}
\end{array}\right.
$$

where $z \in \mathbb{R}^{n}$ is the state of this full-order observer, $\hat{x} \in \mathbb{R}^{n}$ is the estimated state vector and $N, G, L, M, E$ are design matrices for unknown input decoupling goal and other required performances. For simplicity $g(\hat{x})$ is shown as $\hat{g}$ from now on. The parameters of the C-UIO can be obtained using [15]:

$$
\left\{\begin{aligned}
N & =M A-K C \\
G & =M B \\
L & =K(I+C E)-M A E \\
M & =I+E C \\
M D & =0 .
\end{aligned}\right.
$$

It is assumed that $\operatorname{rank}(C D)=\operatorname{rank}(D)$ and the pair $(C, M A)$ is detectable. Using the last equation in (3), $E$ can be obtained as

$$
E=-D(C D)^{+}+Y\left(I-(C D)(C D)^{+}\right),
$$

in which, $(C D)^{+}$is defined as $(C D)^{+}=\left((C D)^{T}(C D)\right)^{-1}(C D)^{T}$ and $\mathrm{Y}$ is a free tunable parameter that can be used to improve the performance, and $K$ is a chosen such that $N$ is Hurwitz [9]. Note that the second term in (4) is such that $\left(I-(C D)(C D)^{+}\right)(C D)=0$ and it generalizes the special solution $-D(C D)^{+} 42$.

Define the estimation error as

$$
e=x-\hat{x} .
$$


As a result

$$
\begin{aligned}
\dot{e} & =\dot{x}-\dot{\hat{x}} \\
& =\dot{x}-\dot{z}+E C \dot{x} \\
& =(I+E C) \dot{x}-\dot{z}=M \dot{x}-\dot{z} .
\end{aligned}
$$

Substituting $\dot{x}$ and $\dot{z}$ from equations (1) and (2) results in

$$
\begin{aligned}
\dot{e} & =M[(A+\Delta A) x+(B+\Delta B) u+D v+g]-N z-G u-L C x-M \hat{g} \\
& =(M A+M \Delta A-L C) x+(M B+M \Delta B-G) u+M D v+M g \\
& -N z-M \hat{g} .
\end{aligned}
$$

Using equations in (3) and replacing $z=\hat{x}+E C x$ one has

$$
\begin{aligned}
\dot{e} & =(M A+M \Delta A-K C-K E C E+M A E C-N E C) x+M \Delta B u \\
& +M(g-\hat{g})-N \hat{x} \\
& =[(M A-K C)+M \Delta A+(M A-K C) E C-N E C)] x+M \Delta B u \\
& +M(g-\hat{g})-N \hat{x} .
\end{aligned}
$$

replacing $N=M A-K C$ results in

$$
\dot{e}=[N+M \Delta A] x+M \Delta B u+M(g-\hat{g})-N \hat{x}
$$

which leads to the continuous error dynamics

$$
\dot{e}=N e+M \Delta A x+M \Delta B u+M(g-\hat{g}) .
$$

Now it it possible to find the gain $K$ such that the error dynamics $(9)$ is robustly stable and satisfies the following requirement

$$
\sup _{\Delta A \in \Gamma_{1}, \Delta B \in \Gamma_{2}} \frac{\|e\|_{2}}{\left\|w_{d}\right\|_{2}}<\lambda,
$$

in which $\lambda>0$ and $w_{d}=\left[x^{T}, u^{T}, v^{T}\right]^{T}$. In the next section, the reset theory is used to introduce a nonlinear observer which can reduce the $\mathcal{L}_{2}$ norm and the settling time of the estimation error. 


\section{Reset UIO}

In this part, reset action is added to the $\mathrm{C}$-UIO to improve the performance of the observer. Thus, the R-UIO can be formulated as

$$
\begin{aligned}
& \left\{\begin{array}{l}
\dot{z}=N z+G u+L y+M \hat{g} \\
\hat{x}=z-E y \\
\hat{y}=C \hat{x}
\end{array}\right\} \text { if } e \in \mathcal{F} \\
& \left\{\begin{array}{l}
z^{+}=\left(M-A_{R} E C\right) z-\left(I-A_{R}\right) M E y \\
\hat{x}^{+}=z^{+}-E y
\end{array}\right\} \text { if } e \in \mathcal{J},
\end{aligned}
$$

in which $A_{R}$ is the after reset matrix, $\mathcal{F}=\left\{e \in \mathbb{R}^{n} \mid e^{T} C^{T} F C e \geq 0\right\}$ is the flow set and $\mathcal{J}=\left\{e \in \mathbb{R}^{n} \mid e^{T} C^{T} F C e \leq 0\right\}$ is the jump set and a jump happens as soon as $e \in \mathcal{J}$. It's worth noting that $F$ and $A_{R}$ will be obtained by solving some inequalities.

For the discrete error dynamics one has

$$
\begin{aligned}
e^{+} & =x-\hat{x}^{+} \\
& =x-z^{+}+E y=(I+E C) x-z^{+}
\end{aligned}
$$

substituting $z^{+}$from (11) results in

$$
e^{+}=x-\left(M-A_{R} E C\right) z+\left(I-A_{R}\right) M E y+E y
$$

using $z=\hat{x}+E C x$ implies that

$$
e^{+}=x-\left(M-A_{R} E C\right)(\hat{x}+E y)+\left(I-A_{R}\right) M E y+E y
$$

simplifying the equation leads to

$$
e^{+}=M e+A_{R} E C \hat{x}-A_{R} E C x
$$

adding and subtracting $A_{R} e, e^{+}$can be obtained as

$$
\begin{aligned}
e^{+} & =M e-A_{R}(I+E C) e+A_{R} e \\
& =\left(A_{R}-A_{R} M+M\right) e .
\end{aligned}
$$

Therefore, defining $H=A_{R}-A_{R} M+M$, the error dynamics can be written as

$$
\left\{\begin{aligned}
\dot{e} & =N e+M \Delta A x+M \Delta B u+M(g-\hat{g}) & & \text { if } e \in \mathcal{F} \\
e^{+} & =H e & & \text { if } e \in \mathcal{J} .
\end{aligned}\right.
$$

Based on reset error dynamics the following theorem on the convergence of R-UIO can be stated: 
Theorem 1. For the system (1) and the observer (11), if there exist symmetric matrices $P>0, F$, matrices $Q, \bar{K}$ and positive scalars $\varepsilon_{1}, \varepsilon_{2}, \lambda, \gamma_{f}, \tau_{f}, \tau_{j}$ and $0<\gamma_{j} \leq 1$ such that

$$
\min _{P, Q, F, A_{R}, \bar{K}, \varepsilon_{1}, \varepsilon_{2}, \gamma_{f}, \gamma_{j}, \tau_{f}, \tau_{j}} \lambda
$$

, subject to

$$
\begin{aligned}
& {\left[\begin{array}{ccccccc}
\Omega_{11} & 0 & 0 & 0 & 0 & 0 & 0 \\
0 & \Omega_{22} & 0 & 0 & P M T_{a} & P M T_{b} & \gamma \sigma P \\
0 & 0 & \Omega_{33} & 0 & 0 & 0 & 0 \\
0 & 0 & 0 & -\lambda^{2} I & 0 & 0 & 0 \\
0 & T_{a}^{T} M^{T} P & 0 & 0 & -\varepsilon_{1} I & 0 & 0 \\
0 & T_{b}^{T} M^{T} P & 0 & 0 & 0 & -\varepsilon_{2} I & 0 \\
0 & \gamma \sigma P & 0 & 0 & 0 & 0 & -I
\end{array}\right]<0} \\
& {\left[\begin{array}{c}
\gamma_{j} P+\tau_{j} F \\
Q-Q M+P M
\end{array}\right] \geq 0,} \\
& H^{T} F H+\tau_{w} C^{T} F C>0,
\end{aligned}
$$

in which

$$
\begin{aligned}
& \Omega_{11}=\varepsilon_{1} N_{a}^{T} N_{a}-\lambda^{2} I \\
& \Omega_{22}=P M A-\bar{K} C+(P M A-\bar{K} C)^{T}+2 I+\gamma_{f} P+\tau_{f} C^{T} F C \\
& \Omega_{33}=\varepsilon_{2} N_{b}^{T} N_{b}-\lambda^{2} I .
\end{aligned}
$$

The error dynamics (16) is robustly stable and has a $\mathcal{L}_{2}$ gain from $w_{d}$ to $e$ which is smaller than $\lambda$ and the optimal gain $K$ can be computed as $K=$ $P^{-1} \bar{K}$.

To prove the theorem, some useful lemmas are presented first.

Lemma 1. Majoration Lemma [43].

Let $X, Y$ and $F(t)$ be real matrices of appropriate dimensions. Then, for any $\varepsilon>0$ and $F(t)^{T} F(t) \leq I$ we have

$$
X F(t) Y+Y^{T} F^{T}(t) X^{T} \leq \varepsilon X X^{T}+\varepsilon^{-1} Y^{T} Y .
$$

Lemma 2. Schur complement 44] 
Let $Q \leq 0, S$, and $R$ be given matrices. The following statements are equivalents

$$
\begin{array}{r}
{\left[\begin{array}{cc}
Q & R \\
R^{T} & S
\end{array}\right] \leq 0,} \\
Q-R S^{-1} R^{T} \leq 0 .
\end{array}
$$

Lemma 3. If the nonlinear function g satisfies the Assumption 1, then the following inequality holds:

$$
2 e^{T} P M(g-\hat{g}) \leq \gamma^{2} \sigma^{2} e^{T} P P e+e^{T} e,
$$

in which $\sigma$ is the largest singular value of $M$ [45].

Proof. Consider the Lyapunov function $V=e^{T} P e$ where $P=P^{T}>0$. The error dynamics $(16)$ is robustly stable and satisfies the condition (10) if

$$
\left\{\begin{array}{rlrl}
\dot{V} & <-\gamma_{f} V+\lambda^{2} u^{T} u+\lambda^{2} x^{T} x+\lambda^{2} v^{T} v-e^{T} e & \text { if } e \in \mathcal{F} \\
V^{+} \leq \gamma_{j} V & \text { if } e \in \mathcal{J} .
\end{array}\right.
$$

Derivation of $V$ can be obtained as follows:

$$
\begin{aligned}
\dot{V} & =\dot{e}^{T} P e+e^{T} P \dot{e} \\
& =(N e+M \Delta A x+M \Delta B u+M(g-\hat{g}))^{T} P e \\
& +e^{T} P(N e+M \Delta A x+M \Delta B u+M(g-\hat{g}))^{T} .
\end{aligned}
$$

Using equation (3), Assumption 2 and change of variable $\bar{K}=P K$ one has

$$
\begin{aligned}
\dot{V} & =e^{T}\left((P M A-\bar{K} C)^{T}+P M A-\bar{K} C\right) e+2 e^{T} P M T_{a} \Sigma_{a} N_{a} x \\
& +2 e^{T} P M T_{b} \Sigma_{b} N_{b} u+2 e^{T} P M(g-\hat{g}) .
\end{aligned}
$$

Applying Lemma 1 and 3 lead to

$$
\begin{aligned}
\dot{V} & \leq e^{T}\left((P M A-\bar{K} C)^{T}+P M A-\bar{K} C\right) e+\varepsilon_{1}^{-1} e^{T} P M T_{a}\left(P M T_{a}\right)^{T} e \\
& +\varepsilon_{1} x^{T} N_{a}^{T} N_{a} x+\varepsilon_{2}^{-1} e^{T} P M T_{b}\left(P M T_{b}\right)^{T} e+\varepsilon_{2} u^{T} N_{b}^{T} N_{b} u \\
& +\gamma^{2} \sigma^{2} e^{T} P P e+e^{T} e
\end{aligned}
$$


Therefore, the first condition in (20) holds if

$$
\begin{aligned}
& e^{T}\left((P M A-\bar{K} C)^{T}+P M A-\bar{K} C\right) e+\varepsilon_{1}^{-1} e^{T} P M T_{a}\left(P M T_{a}\right)^{T} e \\
& +\varepsilon_{1} x^{T} N_{a}^{T} N_{a} x+\varepsilon_{2}^{-1} e^{T} P M T_{b}\left(P M T_{b}\right)^{T} e+\varepsilon_{2} u^{T} N_{b}^{T} N_{b} u \\
& +\gamma^{2} \sigma^{2} e^{T} P P e+e^{T} e+\gamma_{f} V-\lambda^{2} u^{T} u-\lambda^{2} x^{T} x-\lambda^{2} v^{T} v+e^{T} e<0
\end{aligned}
$$

is satisfied for all $e$ such that $e^{T} C^{T} F C e \geq 0$. Using S-procedure [44] with a positive constant $\tau_{f}$, the above condition can be written in the matrix form as

$$
\left[\begin{array}{l}
x \\
e \\
u \\
v
\end{array}\right]^{T}\left[\begin{array}{cccc}
\Omega_{11} & 0 & 0 & 0 \\
0 & \Omega_{22}+\Lambda_{22} & 0 & 0 \\
0 & 0 & \Omega_{33} & 0 \\
0 & 0 & 0 & -\lambda^{2} I
\end{array}\right]\left[\begin{array}{l}
x \\
e \\
u \\
v
\end{array}\right]<0
$$

in which

$$
\Lambda_{22}=\varepsilon_{1}^{-1} P M T_{a}\left(M T_{a}\right)^{T} P+\varepsilon_{2}^{-1} P M T_{b}\left(M T_{b}\right)^{T} P+\gamma^{2} \sigma^{2} P P .
$$

Using Schur complement lemma repeatedly, the condition (25) results in

$$
\left[\begin{array}{ccccccc}
\Omega_{11} & 0 & 0 & 0 & 0 & 0 & 0 \\
0 & \Omega_{22} & 0 & 0 & P M T_{a} & P M T_{b} & \gamma \sigma P \\
0 & 0 & \Omega_{33} & 0 & 0 & 0 & 0 \\
0 & 0 & 0 & -\lambda^{2} I & 0 & 0 & 0 \\
0 & T_{a}^{T} M^{T} P & 0 & 0 & -\varepsilon_{1} I & 0 & 0 \\
0 & T_{b}^{T} M^{T} P & 0 & 0 & 0 & -\varepsilon_{2} I & 0 \\
0 & \gamma \sigma P & 0 & 0 & 0 & 0 & -I
\end{array}\right]<0 .
$$

Similarly, for the discrete error dynamics, one has

$$
V^{+}-\gamma_{j} V \leq 0 .
$$

Using $V^{+}=e^{+} P e^{+}$and substituting $e^{+}$from (16) result in

$$
\begin{gathered}
(H e)^{T} P(H e)-\gamma_{j} e^{T} P e \leq 0 \\
e^{T}\left(H^{T} P H-\gamma_{j} P\right) e \leq 0 .
\end{gathered}
$$


Therefore, it is inferred that

$$
H^{T} P H-\gamma_{j} P \leq 0,
$$

when $e^{T} C^{T} F C e \leq 0$ is satisfied, and with the aid of S-procedure [44 the condition $e^{T} C^{T} F C e \leq 0$ can be added to $(29)$ by using a constant $\tau_{j}>0$ as follows:

$$
H^{T} P H-\gamma_{j} P-\tau_{j} C^{T} F C \leq 0 .
$$

Using the Schur complement lemma the inequality (30) can be rewritten as

$$
\left[\begin{array}{cc}
\gamma_{j} P+\tau_{j} C^{T} F C & H^{T} \\
H & P^{-1}
\end{array}\right] \geq 0
$$

pre and post multiplying (31) by

$$
\left[\begin{array}{ll}
I & 0 \\
0 & P
\end{array}\right]
$$

results in

$$
\left[\begin{array}{cc}
\gamma_{j} P+\tau_{j} C^{T} F C & H^{T} P \\
P H & P
\end{array}\right] \geq 0 .
$$

Replacing $H,(32)$ results in

$$
\left[\begin{array}{cc}
\gamma_{j} P+\tau_{j} C^{T} F C & A_{R}^{T} P-M^{T} A_{R}^{T} P+M^{T} P \\
P A_{R}-P A_{R} M+P M & P
\end{array}\right] \geq 0 .
$$

The inequality (33) is not linear since it contains multiplication of unknown parameters $P$ and $A_{R}$. Therefore, using the variable change $Q=P A_{R}$, one gets

$$
\left[\begin{array}{cc}
\gamma_{j} P+\tau_{j} C^{T} F C & (Q-Q M+P M)^{T} \\
Q-Q M+P M & P
\end{array}\right] \geq 0 .
$$

Moreover, for the well-posedness of the system it is required that after a jump, the error trajectory jumps out of the jump set i.e:

$$
\left(e^{+}\right)^{T} F\left(e^{+}\right)>0 \text { if } e^{T} F e \leq 0 .
$$

Thus, using S-procedure, the inequality

$$
H^{T} F H+\tau_{w} F>0
$$

with the constant $\tau_{w}>0$ must holds and this completes the proof. 
Remark 1. It's worth mentioning that the inequality (36) is checked a posteriori, in practice. It means that as $H$ and $F$ are obtained previously in (17a) and $(17 b)$, if there is $\tau_{w}$ such that the inequality (36) holds then the system is well-posed and in this case, the reset will be applied to the system.

Remark 2. To avoid Zeno solution, temporal regularization time can be included in reset dynamics (11). Including an auxiliary variable $\tau$ guarantees that the time interval between two successive jumps is not smaller than $\rho \in$ $\mathbb{R}^{+}$. If $\rho$ tends to infinity, no reset will occur and if it tends to zero, the Zeno phenomenon may happen [38]. In this case, the error dynamics (16) can be considered as follows:

$$
\begin{aligned}
& \left\{\begin{array}{l}
\dot{e}=N e+M \Delta A x+M \Delta B u+M(g-\hat{g}) \\
\dot{\tau}=1
\end{array}\right\} \text { if } e \in \mathcal{F} \vee \tau \leq \rho \\
& \left\{\begin{array}{l}
e^{+}=H e \\
\tau^{+}=0
\end{array}\right\} \text { if } e \in \mathcal{J} \wedge \tau>\rho .
\end{aligned}
$$

Remark 3. Considering the effect of temporal regularization, if the $R$-UIO hits the reset sector and $\tau \leq \rho$, it has to continue flowing until $\tau>\rho$. In this case stability cannot be assured [40]. To deal with this problem, for a very small $\rho$ a slightly inflated flow region can be considered [38].

Remark 4. The flow set $\mathcal{F}$ and the jump set $\mathcal{J}$ are implementable since the errors Ce are available.

\section{State and fault estimation using R-UIO}

The proposed observer can be used to estimate faults. Consider the following system dynamics:

$$
\begin{aligned}
& \dot{x}=(A+\Delta A) x+(B+\Delta B) u+D v+E_{f} f+g(x) \\
& y=C x
\end{aligned}
$$

in which $f \in \mathbb{R}^{r}$ and $E_{f}$ is a known matrix with appropriate dimension. The number of output channels is greater than or equal to the number of fault inputs i.e $p \geq r$. Moreover, it is assumed that $\dot{f} \simeq 0$. Therefore, the system 
(38) can be rewritten as follows:

$$
\left\{\begin{aligned}
{\left[\begin{array}{c}
\dot{x} \\
\dot{f}
\end{array}\right] } & =\left(\left[\begin{array}{cc}
A & E_{f} \\
0_{r \times n} & 0_{r}
\end{array}\right]+\left[\begin{array}{cc}
\Delta A & 0 \\
0_{r \times n} & 0_{r}
\end{array}\right]\right)\left[\begin{array}{l}
x \\
f
\end{array}\right]+\left(\left[\begin{array}{c}
B \\
0_{r \times m}
\end{array}\right]+\left[\begin{array}{c}
\Delta B \\
0_{r \times m}
\end{array}\right]\right) u \\
& +\left[\begin{array}{c}
D \\
0_{r \times d}
\end{array}\right] v+\left[\begin{array}{c}
g(x) \\
0
\end{array}\right] \\
y & =\left[\begin{array}{ll}
C & 0
\end{array}\right]\left[\begin{array}{l}
x \\
f
\end{array}\right]
\end{aligned}\right.
$$

For conciseness, some new variables and matrices are denoted as follows:

$$
\begin{aligned}
& \bar{x}=\left[\begin{array}{c}
x \\
f
\end{array}\right], \bar{A}=\left[\begin{array}{cc}
A & E_{f} \\
0_{r \times n} & 0_{r}
\end{array}\right], \bar{B}=\left[\begin{array}{c}
B \\
0_{r \times m}
\end{array}\right], \\
& \bar{D}=\left[\begin{array}{c}
D \\
0_{r \times d}
\end{array}\right], \bar{C}=\left[\begin{array}{ll}
C & 0
\end{array}\right], \bar{g}(x)=\left[\begin{array}{c}
g(x) \\
0
\end{array}\right], \\
& \Delta \bar{A}=\left[\begin{array}{cc}
\Delta A & 0 \\
0 & 0
\end{array}\right], \Delta \bar{B}=\left[\begin{array}{c}
\Delta B \\
0
\end{array}\right],
\end{aligned}
$$

then system (38) becomes:

$$
\left\{\begin{array}{l}
\dot{\bar{x}}=(\bar{A}+\Delta \bar{A}) \bar{x}+(\bar{B}+\Delta \bar{B}) u+\bar{D} v+\bar{g} \\
y=\bar{C} \bar{x} .
\end{array}\right.
$$

Now, the system (41) is similar to the system (1) and the observer (2) should be designed for this new augmented system. Moreover, the same procedure explained in Theorem 1 can be applied to estimate fault and states. In this regard, the following Theorem is devised.

Remark 5. It is readily concluded that this fault estimation observer design can be extended to a large class of typical faults, i.e. $f^{(n)}(t)=0$. Moreover, this method can be used for slowly time varying faults as well.

Corollary 1. For the augmented system (41), which includes fault as an auxiliary state, the observer (11) can be designed, provided that the conditions in Theorem 1 are satisfied. Then, the error dynamics for the augmented system is robustly stable and has a $\mathcal{L}_{2}$ gain from $w_{d}$ to e which is smaller than $\lambda$. 
Proof. The proof of this theorem is the same as the Theorem 1, provided that the augmented matrices are used.

Remark 6. The existence conditions of UIO for the augmented system should be check, firstly.

Moreover, for tuning the observer gain, the following lemma can be useful.

Lemma 4. The eigenvalues of a given matrix $N \in \mathbb{R}^{n \times n}$ belong to the circular region $D(\alpha, \tau)$ with center $\alpha+j 0$ and radius $\tau$ if and only if there exists a symmetric positive definite matrix $P \in \mathbb{R}^{n \times n}$ such that the following condition holds:

$$
\left[\begin{array}{cc}
-P & P\left(N-\alpha I_{n}\right) \\
* & -\tau^{2} P
\end{array}\right]<0
$$

\section{Simulation}

Consider a well-mixed CSTR in which the following isothermal, liquid-phase, multi-component chemical reaction $\mathcal{A} \rightleftarrows \mathcal{B} \rightarrow \mathcal{C}$ is being carried out [46 48]. The system model can be described as:

$$
\left\{\begin{array}{l}
\dot{x}_{1}=-\left(1+D a_{1}\right) x_{1}+2 D a_{2} x_{2 d} x_{2}+D a_{2} x_{2}^{2} \\
\dot{x}_{2}=D a_{1} x_{1}-\left(1+2 D a_{2} x_{2 d}+2 D a_{3} x_{2 d}\right) x_{2}-\left(D a_{2}+D a_{3}\right) x_{2}^{2}+u \\
\dot{x}_{3}=2 D a_{3} x_{2 d} x_{2}-x_{3}+D a_{3} x_{2}^{2} \\
y_{1}=x_{3} \\
y_{2}=x_{1}
\end{array}\right.
$$

in which $D a_{1}, D a_{2}, D a_{3}$ are system parameters and $x_{1 d}, x_{2 d},, x_{3 d}$ are steadystate value of the system states. This dynamics can be divided into linear and nonlinear part and rewritten as follows:

$$
\begin{aligned}
\dot{x} & =\left[\begin{array}{ccc}
-\left(1+D a_{1}\right) & 2 D a_{2} x_{2 d} & 0 \\
D a_{1} & -\left(1+2 D a_{2} x_{2 d}+2 D a_{3} x 2 d\right) & 0 \\
0 & 2 D a_{3} x_{2 d} & -1
\end{array}\right] x+\left[\begin{array}{l}
0 \\
1 \\
0
\end{array}\right] u \\
& +\left[\begin{array}{c}
D a_{2} x_{2}^{2} \\
-\left(D a_{2}+D a_{3}\right) x_{2}^{2} \\
D a_{3} x_{2}^{2}
\end{array}\right] \\
y & =\left[\begin{array}{lll}
0 & 0 & 1 \\
1 & 0 & 0
\end{array}\right] x .
\end{aligned}
$$


Under assumptions in [46 48] there is unmodeled first-order dynamics and also an error in measuring the molar feed rate. Moreover, assume that there is uncertainty in system parameters $D a_{1}, D a_{2}, D a_{3}$. In addition, suppose that system is subject to external disturbance. Under such a circumstance, the true process is described by

$$
\begin{aligned}
& \dot{x}=(A+\Delta A) x+(B+\Delta B) u+D v+g(x) \\
& y=C x,
\end{aligned}
$$

in which

$$
\begin{aligned}
& \Delta A=\left[\begin{array}{ccc}
-\Delta D a_{1} & 2 \Delta D a_{2} x_{2 d} & 0 \\
\Delta D a_{1} & \left.-2 \Delta D a_{2} x_{2 d}-2 \Delta D a_{3} x 2 d\right) & 0 \\
0 & 2 \Delta D a_{3} x_{2 d} & 0
\end{array}\right] \\
& \Delta B=\left[\begin{array}{l}
0 \\
b \\
0
\end{array}\right], D=\left[\begin{array}{c}
1 \\
0 \\
0
\end{array}\right], g(x)=\left[\begin{array}{c}
D a_{2} \\
-\left(D a_{2}+D a_{3}\right) \\
D a_{3}
\end{array}\right] x_{2}^{2} .
\end{aligned}
$$

The nominal values for the various constants are $D a_{1}=3.0, D a_{2}=0.5$, and $D a_{3}=1$. The steady-state values of the states are $x_{1 d}=0.3467, x_{2 d}=$ $0.8796, x_{3 d}=0.8796$ and $b=0.3$.

Consequently, the final nominal dynamic model is found accordingly as follows:

$$
\begin{aligned}
& \dot{x}=\left[\begin{array}{ccc}
-4 & 0.8796 & 0 \\
3 & -3.6388 & 0 \\
0 & 1.7592 & -1
\end{array}\right] x+\left[\begin{array}{l}
0 \\
1 \\
0
\end{array}\right] u+\left[\begin{array}{l}
1 \\
0 \\
0
\end{array}\right] v+\left[\begin{array}{c}
0.5 \\
-1.5 \\
1
\end{array}\right] x_{2}^{2} \\
& y=\left[\begin{array}{lll}
1 & 0 & 0 \\
0 & 0 & 1
\end{array}\right] x .
\end{aligned}
$$

It is supposed that there is a 10 percent variation in the system parameters from the nominal value. Therefore, the uncertainty matrices can be described as follows:

$$
\begin{aligned}
& T_{a}=I_{3}, \quad N_{a}=0.1\left[\begin{array}{ccc}
-D a_{1} & 2 D a_{2} x_{2 d} & 0 \\
D a_{1} & -2 D a_{2} x_{2 d}-2 D a_{3} x 2 d & 0 \\
0 & 2 D a_{3} x_{2 d} & 0
\end{array}\right] \\
& T_{b}=B, \quad N_{a}=0.1 b .
\end{aligned}
$$


Moreover, $\Sigma_{a}$ and $\Sigma_{b}$ can be chosen as $\sin (t) I_{3}$ and $\sin (t)$ respectively. The input $u$ is a step with amplitude 1 and the disturbance is $v=\sin (5 t)$.

Now, to obtain the unknown parameters, the R-UIO should be designed by solving the inequalities $17 \mathrm{a}$ ) and $(17 \mathrm{~b}$ ) of Theorem 1 . It is worth noting that, $\gamma_{f}, \gamma_{j}, \tau_{f}$ and $\tau_{j}$ are unknown and result in multiplication of parameters. Therefore, to solve these inequalities, a change of variable is used to remove one of them. Consider $\tau_{j} F=\bar{F}$ thus, $\tau_{f} F$ can be replaced with $\frac{\tau_{f}}{\tau_{j}} \bar{F}=\overline{\tau_{f}} \bar{F}$. It is the same as letting $\tau_{j}=1$ and solving the inequalities. Since an analytical solution for the optimal value of the unknown parameters can't be obtained, a numerical approach is used to find a suboptimal solution.

One way to deal with the aforementioned problem is to consider a grid for $\gamma_{f}, \gamma_{j}$ and $\tau_{j}$, then the inequalities are solved at each point of the grid to obtain a feasible solution. An alternative method is to suppose, there is no jump and uncertainty in the model firstly. Therefore only inequality (17a) should be solved to obtain the $\gamma_{f}$ and $\tau_{f}$. In this case, the unknown parameters $\gamma_{f}$ and $\tau_{f}$ can be obtained by just griding in two dimensions. Now the $\gamma_{f}$ and $\tau_{f}$ can be fixed and make a one-dimensional grid to obtain $\gamma_{j}$ and try to minimize $\lambda$.

Remark 7. The matrix $F$ should be chosen such that it is neither positive definite nor negative definite in order to represent a sector.

\subsection{State estimation}

First, the system dynamics (1) without any fault is considered. Initial condition of the system and the observer are $x=[0.3,0.3,0.5]^{T}$ and zero respectively. Following the previously explained procedure, the parameters can be obtained as $\gamma_{f}=1.2, \gamma_{j}=1, \tau_{f}=60$ and let $\gamma=0.1$. Choosing

$$
Y=\left[\begin{array}{cc}
0.3368 & -0.3454 \\
-0.3892 & 1.1948 \\
-0.4291 & 0.8448
\end{array}\right]
$$

and applying the Theorem 1 to the system, the unknown parameters can be obtained as:

$$
P=\left[\begin{array}{ccc}
1.1107 & 0.0228 & -0.0275 \\
0.0228 & 0.4874 & 0.0386 \\
-0.0275 & 0.0386 & 1.1070
\end{array}\right], F=\left[\begin{array}{cc}
-0.0178 & 0.0296 \\
0.0296 & 0.0334
\end{array}\right]
$$




$$
\begin{gathered}
A_{R}=\left[\begin{array}{ccc}
0.0000 & -0.8655 & 0.0003 \\
0.0468 & 1.2551 & -0.0099 \\
0.0000 & -1.4822 & 0.0141
\end{array}\right], K=\left[\begin{array}{cc}
1.3611 & 2.9107 \\
2.1631 & 4.0027 \\
0.9769 & -0.0636
\end{array}\right], \\
N=\left[\begin{array}{ccc}
-2.9107 & 0.5925 & -1.6979 \\
-1.0027 & -4.3235 & -1.7739 \\
0.0636 & 1.0043 & -1.5478
\end{array}\right]
\end{gathered}
$$

Using the proposed observer, the state estimation and the estimation errors in both C-UIO and R-UIO are shown in the Figure 1. This Figure shows that state estimation using R-UIO can be more accurate and faster. Moreover, it can be seen that utilizing the proposed method can decrease the undershoot. In addition, R-UIO shows more robust behavior and performance in the presence of uncertainty. It means that R-UIO not only robustly estimates the states, but also improves the estimation in the presence of norm bounded uncertainty resulting from the modeling and disturbances. It is worth mentioning that after the first reset in about $t=0.15 \mathrm{~s}$ the state estimation error jump toward zero suddenly. This shows the proper choice of after reset value. As a result, it can be seen that a direct consequence of the jump in the estimations is reduction of $\mathcal{L}_{2}$ norm of error and settling time. Table 1 shows the $\mathcal{L}_{2}$ norm and the settling time $(5 \%)$ of the estimation error. It can be seen that the devised R-UIO outperforms the C-UIO and improves the results in both $\mathcal{L}_{2}$ norm and the settling time of estimation error. The last row in Table 1 presents the percentage of improvement in performance measures using $\mathrm{R}-\mathrm{UIO}$ with respect to $\mathrm{C}-\mathrm{UIO}$.

Table 1: $\mathcal{L}_{2}$ norm and Settling time comparison

\begin{tabular}{|c|c|c|c|}
\hline Method & R-UIO & C-UIO & Improvement $(\%)$ \\
\hline$T_{s t l}(s)$ & 0.2113 & 1.6222 & 86.9745 \\
\hline$\sqrt{\int_{0}^{\infty} e^{T} e d t}$ & 0.2098 & 0.3239 & 35.2466 \\
\hline
\end{tabular}

Furthermore, a statistical analysis has been done to demonstrate the effectiveness of the proposed method. Regarding this, a Monte-Carlo simulation with the different initial conditions has been run and the improvement in the performance indices are shown in Table 2. As can been seen, for the $63.1 \%$ of the initial conditions the $\mathcal{L}_{2}$ norm improved using reset. Besides, for the $68.6 \%$ of the samples the settling time reduces. Moreover, the average improvement is $7.7 \%$ and $5.8 \%$. 


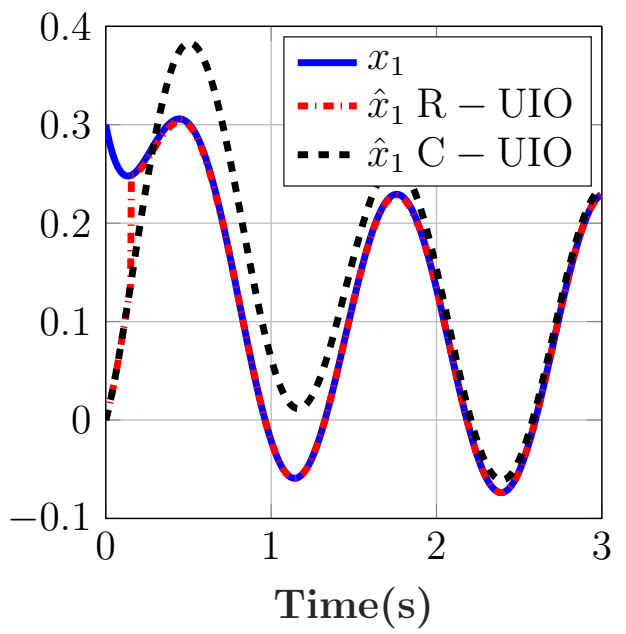

(a) 1st state of the plant

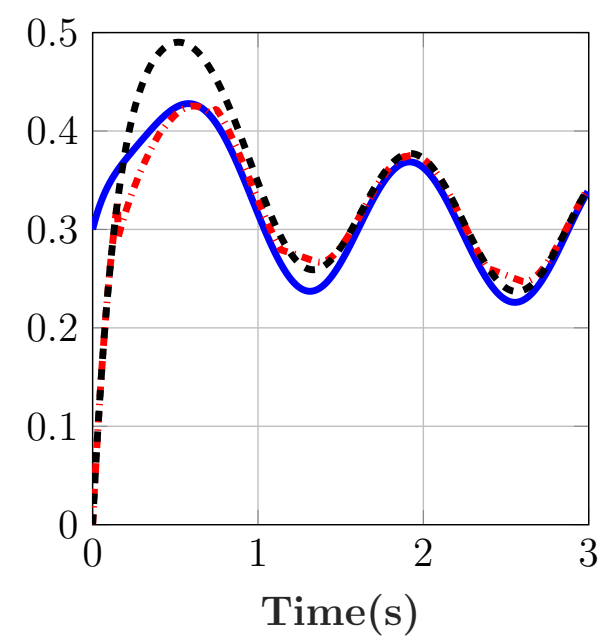

(c) 2nd state of the plant

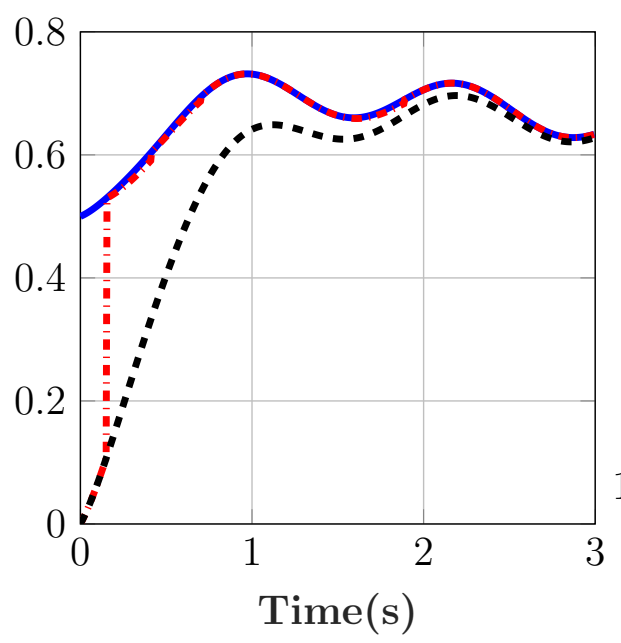

(e) 3rd state of the plant

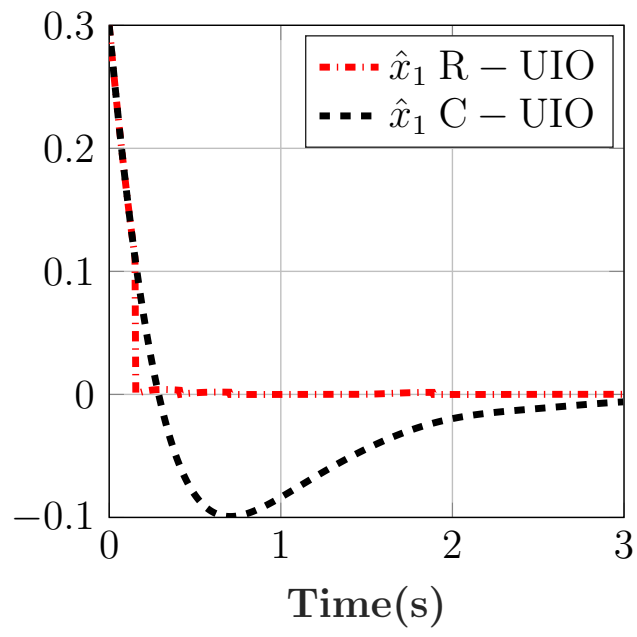

(b) 1st state estimation error

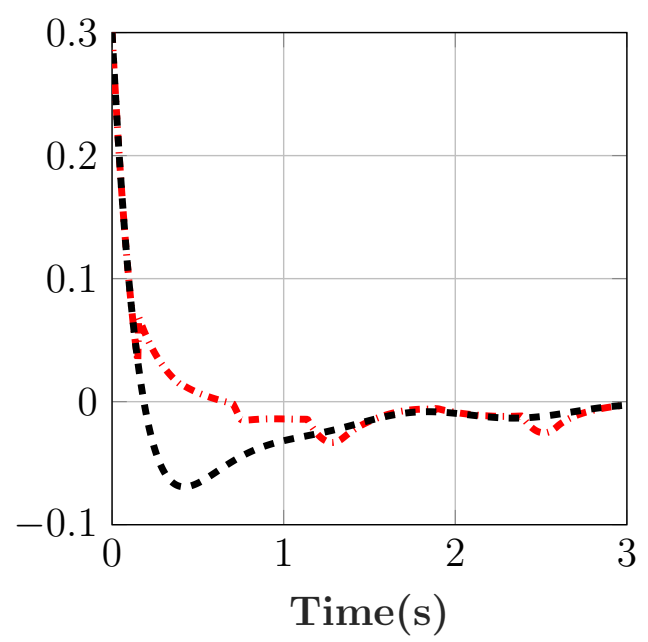

(d) 2nd state estimation error

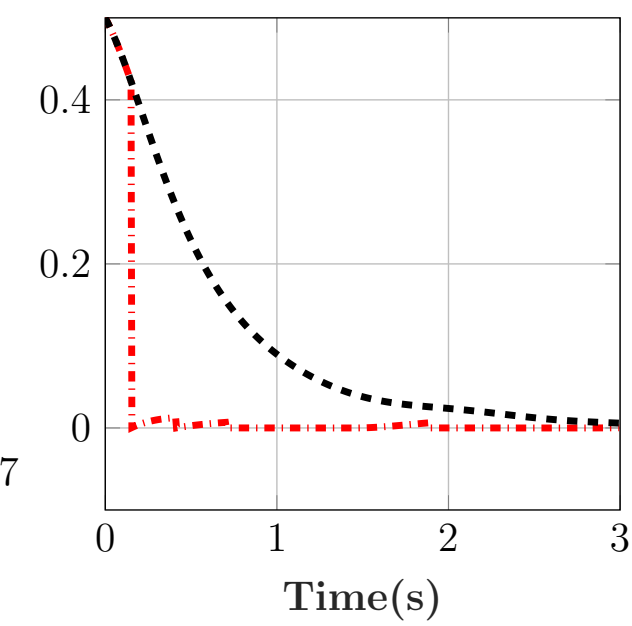

(f) 3rd state estimation error

Figure 1: State estimation and estimation error 
Table 2: Result of Monte-Carlo simulation

\begin{tabular}{|c|c|c|c|}
\hline \multicolumn{2}{|c|}{ Average } & \multicolumn{2}{c|}{ Total } \\
\hline$\|e\|_{2}$ & $T_{\text {stl }}$ & $\|e\|_{2}$ & $T_{\text {stl }}$ \\
\hline 7.7 & 5.8 & 63.1 & 68.6 \\
\hline
\end{tabular}

\subsection{State and fault estimation}

Now consider the CSTR dynamics in (44), which is in the general form of (45). Suppose that there is an actuator fault in the system model with $\overline{E_{f}}=B$. Hence, the faulty model can be described in the general form of (38). The fault is considered as an abrupt fault as follows:

$$
f(t)=\left\{\begin{array}{cc}
1 & 3 \leq t \leq 7 \\
0 & \text { else. }
\end{array}\right.
$$

Constructing the augmented system and applying Theorem 1 along with the Lemma 4 with $\alpha=-3.5, \tau=1.5$, the unknown parameters can be obtained as: $\gamma_{f}=1, \gamma_{j}=1, \tau_{f}=2$ and let $\gamma=0.1$. Choosing

$$
Y=\left[\begin{array}{ll}
-0.6537 & -0.2857 \\
-1.2294 & -0.4624 \\
-0.2710 & -0.4098 \\
-0.9000 & -0.5035
\end{array}\right]
$$

and applying the Theorem 1 to the system, the unknown parameters can be obtained as:

$$
\begin{gathered}
P=\left[\begin{array}{cccc}
324.5537 & 15.2064 & 30.9417 & 5.1134 \\
15.2064 & 386.8132 & -31.4134 & -88.4059 \\
30.9417 & -31.4134 & 275.6123 & -10.5633 \\
5.1134 & -88.4059 & -10.5633 & 22.1028
\end{array}\right], F=\left[\begin{array}{cc}
6.7706 & 28.7999 \\
28.7999 & -38.7831
\end{array}\right] \\
A_{R}=\left[\begin{array}{cccc}
-0.0000 & 0.1213 & 0.0938 & 0.3797 \\
1.0737 & -1.1097 & -0.6701 & -2.5316 \\
0.0000 & -0.2982 & -0.0908 & -0.8244 \\
4.5259 & -4.7495 & -2.9348 & -13.3870
\end{array}\right], K=\left[\begin{array}{cc}
4.4974 & -0.4310 \\
-0.5205 & 7.7351 \\
-0.7167 & 5.0409 \\
-15.6523 & 32.8381
\end{array}\right], \\
N=\left[\begin{array}{cccc}
-4.4974 & -0.5026 & 0.7167 & 0 \\
3.5205 & -4.4523 & -7.2727 & 1.0000 \\
0.7167 & 1.0383 & -5.6311 & 0 \\
15.6523 & -0.8858 & -32.3346 & 0
\end{array}\right] .
\end{gathered}
$$


Table 3: Fault estimation $\mathcal{L}_{2}$ norm and Settling time comparison

\begin{tabular}{|c|c|c|c|c|}
\hline Method & R-UIO & C-UIO & R-PIO & Improvement(\%) \\
\hline$T_{\text {stl }}(s)$ & 1.50 & 2.30 & 2.2 & 34.78 \\
\hline$\sqrt{\int_{0}^{\infty} e^{T} e d t}$ & 0.94 & 1.16 & 1.24 & 18.97 \\
\hline
\end{tabular}

The result of state and fault estimation with the initial condition $\bar{x}_{0}=$ $[1,0.1,0.23,0.1]^{T}$ can be seen in the Figure 2 and 3 respectively. In Figure 2 the estimation of states subject to fault and disturbance is presented. In this figures, the results of our method is compared with the C-UIO and proportional-integral reset observer (R-PIO) [38] and [39]. The results show that state estimation using R-UIO can result in smaller overshoot and faster estimation compared to the C-UIO and R-PIO. In addition, the Figure 3 depicts the fault estimation and the result of the proposed R-UIO is compared with the other methods. It can be seen that the R-UIO fault estimation outperforms the C-UIO and R-PIO and estimates the states and fault more rapidly and accurately. Besides, it can be deduced that despite the presence of modeling uncertainties, R-UIO can estimate the fault and states better than the other mentioned methods which in turn demonstrates the robust stability of the CSTR error dynamics. Just like the state estimation in previous section, in this case, due to proper choice of reset law and after reset value, state estimation error jumps toward zero after the first reset moment. Moreover, in Table 3 a comparison of the R-UIO, C-UIO and R-PIO in estimating the fault is presented. It shows that using the devised method can result in about $35 \%$ and $19 \%$ improvement in settling time and $\mathcal{L}_{2}$ norm of the estimation error with respect to C-UIO.

It is worth mentioning that for reset-based approaches, the choice of the reset law and the after reset value is the key stage. It is not always evident how to choose them. Care should be taken to choose these parameters.

To wrap it up, from the results, three important conclusions can be revealed:

a) Resetting action can improve the fault and state estimation.

b) R-UIO provides estimation with better transient response than the other methods.

c) The improvement in the fault estimation using R-UIO in comparison with the C-UIO is about $35 \%$ in settling time $19 \%$ in $\mathcal{L}_{2}$ norm of the estimation error. 


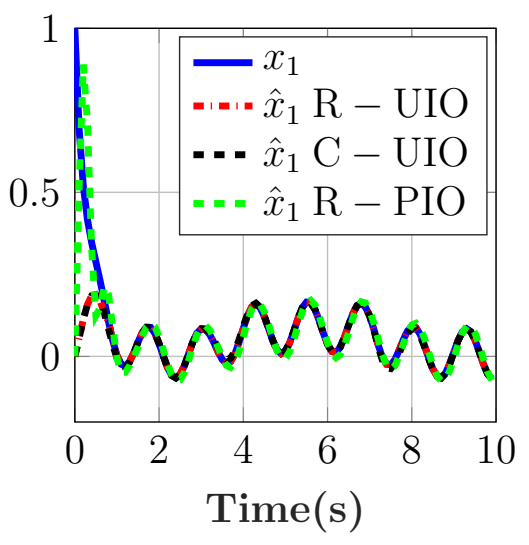

(a) 1st state of the plant

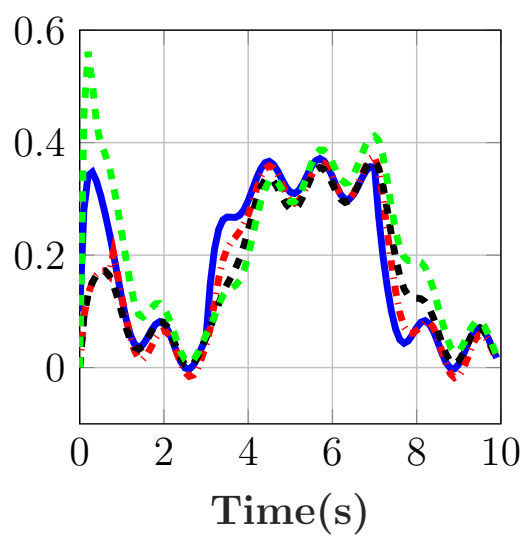

(c) 2nd state of the plant

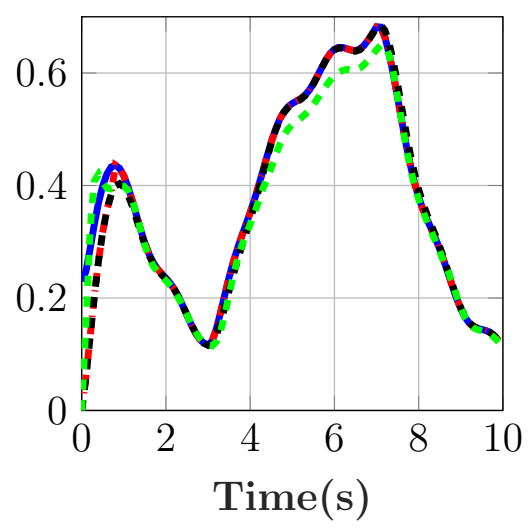

(e) 3rd state of the plant

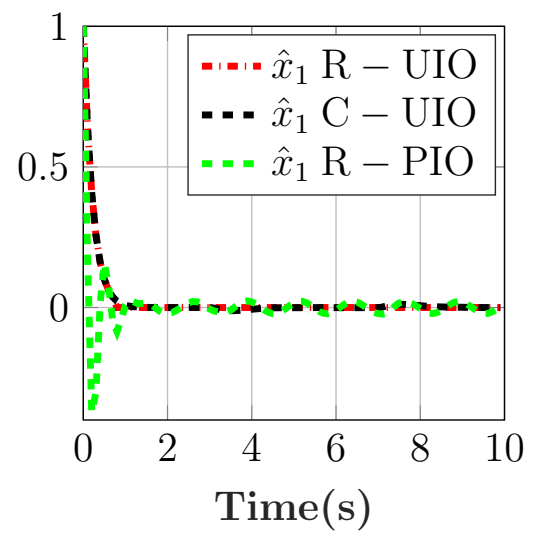

(b) 1st state estimation error

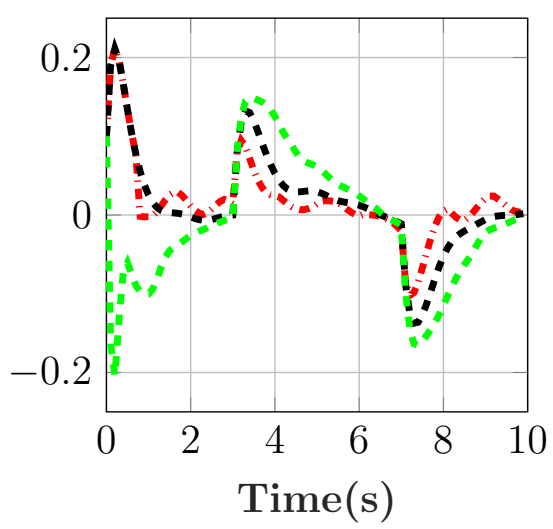

(d) 2nd state estimation error

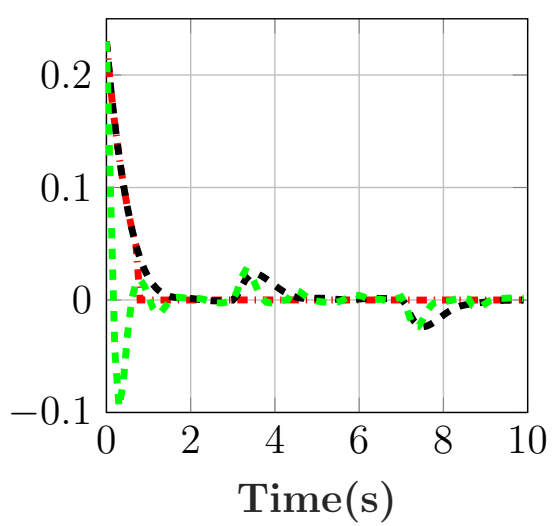

(f) 3rd state estimation error

Figure 2: State estimation and estimation error 


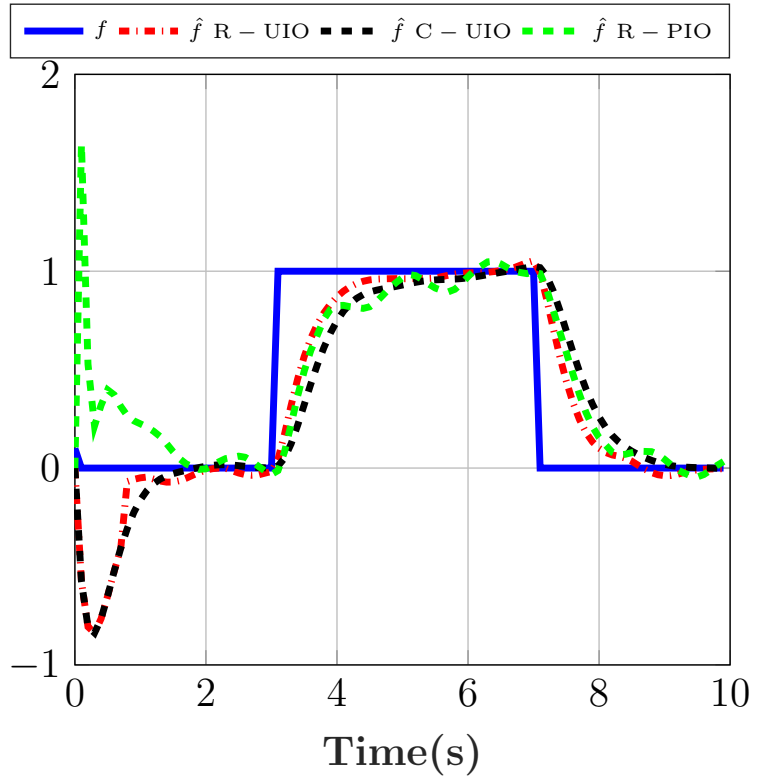

(a) Fault

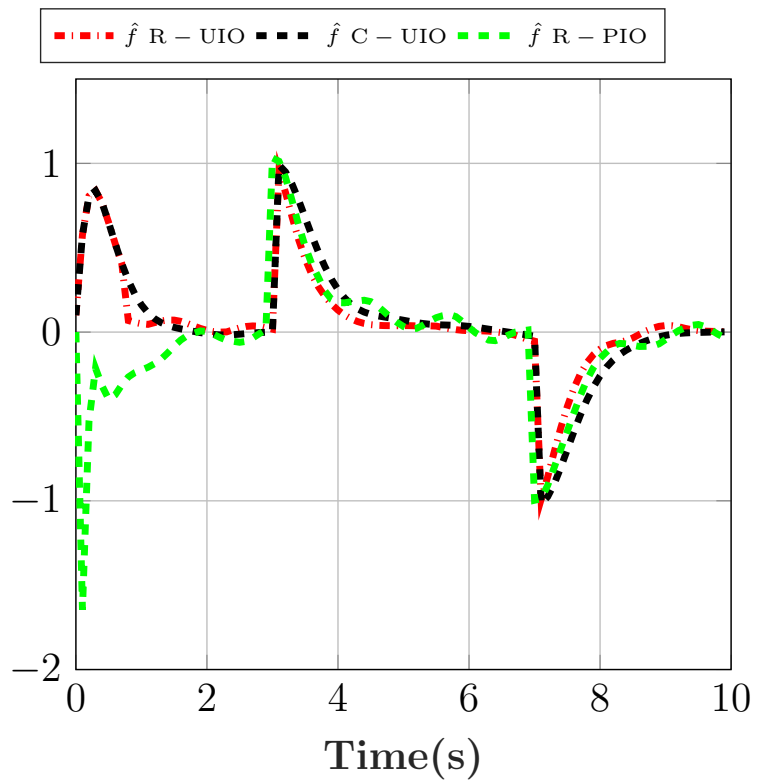

(b) Fault estimation error

Figure 3: Fault estimation and estimation error 


\section{Conclusion}

In this paper, optimal Reset Unknown Input Observer for a class of nonlinear uncertain systems was proposed. In this observer, the states are reset to an optimal value based on a $H_{\infty}$ optimization problem. The devised observer can be used to estimate fault and states simultaneously by considering fault as an auxiliary state and constructing the augmented system. Robust stability of the proposed method is addressed by utilizing a CSTR model as a practical example. It is shown that using the reset in the UIO can decrease the $\mathcal{L}_{2}$ cost and the settling time of the estimation error. 


\section{References}

[1] T. Park and D. Kim. Design of unknown input observers for linear systems with unmatched unknown inputs. Transactions of the Institute of Measurement and Control, 36(3):399-410, 2014.

[2] P.P Menon and C. Edwards. A sliding mode observer for monitoring and fault estimation in a network of dynamical systems. International Journal of Robust and Nonlinear Control, 24(17):2669-2685, 2014.

[3] J. Yang, J. Su, S. Li, and X. Yu. High-order mismatched disturbance compensation for motion control systems via a continuous dynamic sliding-mode approach. IEEE Transactions on Industrial Informatics, 10(1):604-614, 2014.

[4] W. Kim and C.C Chung. Robust output feedback control for unknown non-linear systems with external disturbance. IET Control Theory 8 Applications, 10(2):173-182, 2016.

[5] X. Liu, X. Gao, and J. Han. Observer-based fault detection for highorder nonlinear multi-agent systems. Journal of the Franklin Institute, 353(1):72-94, 2016.

[6] F. Yang and R.W Wilde. Observers for linear systems with unknown inputs. IEEE Transactions on Automatic Control, 33(7):677-681, 1988.

[7] M. Darouach, M. Zaadzinski, and S. Xu. Full-order observers for linear systems with unknown inputs. IEEE Transactions on Automatic Control, 39(3):606-609, 1994.

[8] M.S. Chen and C.C Chen. Unknown input observer for linear nonminimum phase systems. Journal of the Franklin Institute, 347(2):577$588,2010$.

[9] J. Chen and R.J. Patton. Robust Model-Based Fault Diagnosis for Dynamic Systems. The International Series on Asian Studies in Computer and Information Science. Springer US, 2012.

[10] D. Koenig and S. Mammar. Design of a class of reduced order unknown inputs nonlinear observer for fault diagnosis. In American Control Conference., volume 3, pages 2143-2147. IEEE, 2001. 
[11] M. Corless and J. Tu. State and input estimation for a class of uncertain systems. Automatica, 34(6):757-764, 1998.

[12] X. Gao, X. Liu, and J. Han. Reduced order unknown input observer based distributed fault detection for multi-agent systems. Journal of the Franklin Institute, 354(3):1464-1483, 2017.

[13] S. Hui and S.H Żak. Stress estimation using unknown input observer. In American Control Conference (ACC), pages 259-264. IEEE, 2013.

[14] S. Mondal, G. Chakraborty, and K. Bhattacharyy. LMI approach to robust unknown input observer design for continuous systems with noise and uncertainties. International Journal of Control, Automation and Systems, 8(2):210-219, 2010.

[15] W. Chen and M. Saif. Unknown input observer design for a class of nonlinear systems: an LMI approach. In American Control Conference, 2006, pages 5-pp. IEEE, 2006.

[16] W. Zhang, H. Su, F. Zhu, and G.M Azar. Unknown input observer design for one-sided lipschitz nonlinear systems. Nonlinear Dynamics, 79(2):1469-1479, 2015.

[17] M. Chadli and H.R. Karimi. Robust observer design for unknown inputs Takagi-Sugeno models. IEEE Transactions on Fuzzy Systems, 21(1):158-164, 2013.

[18] D. Rotondo, M. Witczak, V. Puig, F. Nejjari, and M. Pazera. Robust unknown input observer for state and fault estimation in discretetime Takagi-Sugeno systems. International Journal of Systems Science, 47(14):3409-3424, 2016.

[19] B. Kulcsár, J. Bokor, and J. Shinar. Unknown input reconstruction for lpv systems. International Journal of Robust and Nonlinear Control: IFAC-Affiliated Journal, 20(5):579-595, 2010.

[20] S. Li, H. Wang, A. Aitouche, Y. Tian, and N. Christov. Robust unknown input observer design for state estimation and fault detection using linear parameter. In 13th European Workshop on Advanced Control and Diagnosis, 2017. 
[21] K. Esfandiari, F. Abdollahi, and H.A Talebi. Adaptive near-optimal neuro controller for continuous-time nonaffine nonlinear systems with constrained input. Neural Networks, 93:195-204, 2017.

[22] J.C Clegg. A nonlinear integrator for servomechanisms. Transactions of the American Institute of Electrical Engineers, Part II: Applications and Industry, 77(1):41-42, 1958.

[23] I. Horowitz and P. Rosenbaum. Non-linear design for cost of feedback reduction in systems with large parameter uncertainty. International Journal of Control, 21(6):977-1001, 1975.

[24] Y. Chait and CV Hollot. On Horowitz's contributions to reset control. International Journal of Robust and Nonlinear Control, 12(4):335-355, 2002.

[25] Y. Guo, W. Gui, C. Yang, and L. Xie. Stability analysis and design of reset control systems with discrete-time triggering conditions. Automatica, 48(3):528-535, 2012.

[26] M.A Davó and A. Baños. Reset control of a liquid level process. In Emerging Technologies 83 Factory Automation (ETFA), IEEE 18th Conference on, pages 1-4. IEEE, 2013.

[27] J. Carrasco and E.M Navarro-López. Towards $L_{2}$-stability of discretetime reset control systems via dissipativity theory. Systems $\&$ Control Letters, 62(6):525-530, 2013.

[28] A. Baños, F. Perez, and J. Cervera. Network-based reset control systems with time-varying delays. IEEE Transactions on Industrial informatics, 10(1):514-522, 2014.

[29] A. Baños, J. I Mulero, A.o Barreiro, and M.A Davó. An impulsive dynamical systems framework for reset control systems. International Journal of Control, 89(10):1985-2007, 2016.

[30] M. Fiacchini and I. Morărescu. Constructive necessary and sufficient condition for the stability of quasi-periodic linear impulsive systems. IEEE Transactions on Automatic Control, 61(9):2512-2517, 2016. 
[31] X. Xiao, J.H. Park, and L. Zhou. Stabilization of switched linear singular systems with state reset. Journal of the Franklin Institute, 356(1):237$247,2019$.

[32] G.X. Zhong and G.H. Yang. Asynchronous fault detection and robust control for switched systems with state reset strategy. Journal of the Franklin Institute, 355(1):250-272, 2018.

[33] V. Ghaffari, P. Karimaghaee, and A. Khayatian. Reset law design based on robust model predictive strategy for uncertain systems. Journal of Process Control, 24(1):261-268, 2014.

[34] M. Fiacchini, S. Tarbouriech, and C. Prieur. Exponential Stability for Hybrid Systems with Saturations, chapter 7, pages 179-212. John Wiley \& Sons, Ltd, 2013.

[35] M. Davanipour, A. Khayatian, and M. Dehghani. Optimal adaptive reset control with guaranteed transient and steady state tracking error bounds. Journal of the Franklin Institute, 354(14):5949-5963, 2017.

[36] N. Vafamand and A. Khayatian. Model predictive-based reset gainscheduling dynamic control law for polytopic LPV systems. ISA transactions, 81:132-140, 2018.

[37] D. Paesa, C. Franco, S. Llorente, G. Lopez-Nicolas, and C. Sagues. Reset adaptive observers and stability properties. In 18th Mediterranean Conference on Control \& Automation (MED), pages 1435-1440. IEEE, 2010 .

[38] D. Paesa, A. Banos, and C. Sagues. Optimal reset adaptive observer design. Systems $\&$ Control Letters, 60(10):877-883, 2011.

[39] Z. Meng, W. Zhenhua, W. Yan, and Shen Yi. A fast fault diagnosis method based on reset augmented observer. In 34th Chinese Control Conference (CCC), pages 6291-6296. IEEE, 2015.

[40] I. Hosseini, A. Khayatian, P. Karimaghaee, M. Fiacchini, and M.A. Davo. LMI-Based Reset Unknown Input Observer for State Estimation of Linear Uncertain Systems. IET Control Theory $\& 5$ Applications, MAY 2019 . 
[41] A. Aminzadeh and A. Khayatian. Reset observer design for time-varying dynamics: Application to WIG crafts. Aerospace Science and Technology, 81:32-40, 2018.

[42] M. James. The generalised inverse. The Mathematical Gazette, 62(420):109-114, 1978.

[43] Y. Wang, L. Xie, and C.E. de Souza. Robust control of a class of uncertain nonlinear systems. Systems \& Control Letters, 19(2):139-149, 1992.

[44] S. Boyd and L. Vandenberghe. Convex optimization. Cambridge university press, 2004.

[45] S. Raghavan and J.K. Hedrick. Observer design for a class of nonlinear systems. International Journal of Control, 59(2):515-528, 1994.

[46] C.T. Chen and S.T. Peng. A nonlinear control scheme for imprecisely known processes using the sliding mode and neural fuzzy techniques. Journal of Process Control, 14(5):501-515, 2004.

[47] C.T. Chen and C.S. Dai. Robust controller design for a class of nonlinear uncertain chemical processes. Journal of Process Control, 11(5):469-482, 2001.

[48] V. Ghaffari, S.V. Naghavi, and A.A. Safavi. Robust model predictive control of a class of uncertain nonlinear systems with application to typical CSTR problems. Journal of Process Control, 23(4):493-499, 2013. 